\title{
Computing SS Index of Certain Dendrimers
}

\author{
Weidong Zhao $\mathbb{D},{ }^{1}$ M.C. Shanmukha $\mathbb{D}^{2}{ }^{2}$ A. Usha, ${ }^{3}$ Mohammad Reza Farahani $\mathbb{D}{ }^{4}$ \\ and K.C. Shilpa ${ }^{5}$ \\ ${ }^{1}$ School of Computer Science, Chengdu University, Chengdu, China \\ ${ }^{2}$ Department of Mathematics, Jain Institute of Technology, Davanagere-577003, Karnataka, India \\ ${ }^{3}$ Department of Mathematics, Alliance School of Applied Mathematics, Alliance University, Bangalore-562106, Karnataka, India \\ ${ }^{4}$ Department of Mathematics, Iran University of Science and Technology, Narmak, Tehran 16844, Iran \\ ${ }^{5}$ Department of Computer Science \& Engg, Bapuji Institute of Engineering and Technology, Davanagere-577004, \\ Karnataka, India
}

Correspondence should be addressed to M.C. Shanmukha; mcshanmukha@gmail.com

Received 25 June 2021; Accepted 28 August 2021; Published 25 September 2021

Academic Editor: Huseyin Isik

Copyright $(2021$ Weidong Zhao et al. This is an open access article distributed under the Creative Commons Attribution License, which permits unrestricted use, distribution, and reproduction in any medium, provided the original work is properly cited.

The numerical descriptor gathers the data from the molecular graphs and helps to know the characteristics of the chemical structure known as topological index. The QSAR/QSPR/QSTR studies are benefited with the significant role played by topological indices in the drug design. Topological indices provide the information about the physical/chemical/biological properties of chemical compounds. The Zagreb indices are widely studied because of their extensive usage in chemical graph theory. Inspired by the earlier work on inverse sum indeg index (ISI index), novel topological index known as SS index is introduced and computed for four dendrimer structures. Also, the strong correlation coefficient between SS index and 5 physico-chemical characteristics such as boiling point $(b p)$, molar volume $(m v)$, molar refraction $(m r)$, heats of vaporization $(h v)$, and critical pressure $(c p)$ of 67 alkane isomers have been determined. It is found that newly introduced index has shown good correlation in comparison with three most popular existing indices (ISI index and first and second Zagreb indices). In the last part, the mathematical properties of SS index are discussed.

\section{Introduction and Terminologies}

Every year, large number of new drugs are produced due to the rapid growth of medicine manufacturing. As a result, determining the pharmacological, chemical, and biological characteristics of a substance necessitates a significant amount of effort.

These new medications are becoming increasingly clumsy and clumped. In order to check the performance of new drugs and their side effects, sufficient reagents, equipment, and technicians are needed. However, in lowincome countries, there is an insufficient funding to cover the costs of reagents and equipment needed to calculate biochemical properties. The existing studies have shown that the chemical and pharmacodynamic properties of drugs, as well as their molecular structures, are inextricably related. If we quantify measures of these drug molecular structures with the aim of identifying topological indices, medical and pharmaceutical researchers will be able to understand their therapeutic properties, which can compensate for the shortcomings of medicine and chemical experiments. In this regard, the methods computing topological index are suitable and useful for developing countries, as they can produce accessible biological and medical knowledge about new drugs without the use of chemical experiment hardware. To calculate the characteristics of drug molecules, the PI index, Zagreb index, and eccentric index are used. The number of vertices and edges of a chemical compound counts to the computation of topological indices [1-8].

A topological index is a computational parameter derived from the graph structure mathematically [9-13]. To visualize the relationships between the data sets, graphs are crucial tools which make the concept better understandable. A descriptor that gives the data regarding arrangement of 
atoms in a compound in numerical form of information regarding its shape, branching, and other data of a compound is a topological index.

The significant number of early drug studies suggests that the biomedical and pharmacology properties of drugs, as well as their molecular structures, have a clear inner relationship. Many scientists have developed various indices to quantify the characteristics of drug molecules over the last 40 years. The indices are of great use in the study of pharmacology, toxicology, and chemistry (QSAR/QSPR/ QSTR) [14-16].

Dendrimers are also called "cascade molecules," but this term is not in general use compared to the term dendrimers. In 1978, Fritz Vogtle was the first to bring these nanomolecules into light. Dendrites normally include a unique chemically addressable unit known as focus or core. The usage and popularity of dendrimers have been greatly increased. Since 2005, there have been over 5000 research papers and patents. A second group of the synthesized macromolecules is called arborols. We can say that the molecules of dendrimers are of architectural design. These thoroughly tailored architectural nanomolecules can be functionalized and modify their physico-chemical or biological characteristics.

The hyper-branched macromolecules have three phases in its structural constitution. An atom at the centre of the structure called the core of the dendrimer has some functional properties. Secondly, the branches are ejected out of the core and add on the branches repetitively. Finally, the terminal groups are situated on the surface of the dendritic structure. Dendrimer synthesis is divided into two methods: divergent synthesis and convergent synthesis. It is difficult to synthesize dendrimers using either approach, because the actual reactions require several steps to protect the active site. As a result, it is difficult to manufacture and prohibitively costly to buy. Dendrites have significant applications in biomedical field because of its characteristics, including hyper-branching, well-defined globular structures, outstanding structural uniformity, multivalency, varying chemical constitution, and higher biological compatibility.

In medical field, mathematical modelling is used to analyse the representation of emerging drugs, normally as an undirected graph, such that each vertex depicts an atom and an edge depicts a link between atoms. Every year new drugs are available and needs remarkable work to select the qualities of the emerging drugs. Dendrimers are a good option in the drug design because of its biological characteristics such as polyvalency, self-assembling, electrostatic interactions, chemical stability, low cytotoxicity, and solubility. The remarkable and emerging role of dendritic macromolecules is in therapies of anticancer and image diagnosis.

Various studies have revealed that there is a consistent correlation between the molecular structures of compounds, drugs, and their characteristics. Topological indices are numerical variants that assist researchers in understanding physical properties, chemical interactions, and biological activity [17-21]. Hence, the discussion on topological indices of chemical structures of drugs helps to know the theoretical basis to prepare new drugs. In this study, SS index is defined and computed for porphyrin $\left(D_{n} P_{n}\right)$, propyl ether imine $\left(\mathrm{DPZ}_{n}\right)$, zinc porphyrin (PETIM), and polyethylene amide amine (PETAA) dendrimers [22,23].

In this paper, the notations and terminologies pertaining to the graphs are found in [24].

Definition 1. The oldest and the most studied indices, the first and second Zagreb indices [25], proposed by Gutman and Trinajstić are defined as

$$
\begin{aligned}
& M_{1}(G)=\sum_{v w \varepsilon E(G)}\left(\mathrm{d}_{v}+\mathrm{d}_{w}\right), \\
& M_{2}(G)=\sum_{v w \varepsilon E(G)}\left(\mathrm{d}_{v} \cdot \mathrm{d}_{w}\right) .
\end{aligned}
$$

Definition 2. Vukičević et al. introduced inverse sum indeg index [26] and stated as

$$
\operatorname{ISI}(G)=\sum_{\nu \omega \in E(G)} \frac{\mathrm{d}_{\nu} \cdot \mathrm{d}_{\omega}}{\mathrm{d}_{\nu}+\mathrm{d}_{\omega}}
$$

Definition 3. In this work, a novel invariant known as SS (Shilpa-Shanmukha) index is introduced and studied. This index is defined as follows:

$$
\mathrm{SS}(G)=\sum_{\nu \omega \in E(G)} \sqrt{\frac{\mathrm{d}_{v^{*}} \mathrm{~d}_{\omega}}{\mathrm{d}_{\nu}+\mathrm{d}_{\omega}}} .
$$

Throughout this article, $d_{v}$ and $d_{\omega}$ represent the degrees of vertices $v$ and $\omega$, respectively.

\section{Chemical Applicability of the SS Index through QSPR Analysis}

Here, we discussed the proposed topological index known as SS index to study the physico-chemical properties, namely, $b p, m v, m r, h v$, and $c p$ of 67 alkanes ranging from $n$-butanes to nonanes. The 5 physico-chemical properties of 67 alkane isomers can be found in [27] and Table 1 represents the computed values of four topological indices (SS, ISI, $M_{1}$, and $\mathrm{M}_{2}$ ) of 67 alkane isomers. The 5 characteristics of alkane isomers are correlated with SS index and it is found that SS index has shown good correlation with all the 5 properties compared to the existing three most popular indices $M_{1}, M_{2}$, and ISI considered in the study. The SS index is plotted against each of the 5 properties of alkane isomers which is depicted in Figure 1.

Regression model for properties of alkane isomers.

The linear regression model is given by

$$
P=m(\mathrm{TI})+c
$$

where $P$ is the physical property and TI is the topological index. Equation (4) results in the following linear regression models for various properties with SS index. 
TABLE 1: The values of various topological indices of alkanes.

\begin{tabular}{|c|c|c|c|c|c|}
\hline SI. No. & Alkane & SS (G) & ISI (G) & $M_{1}(G)$ & $M_{2}(G)$ \\
\hline 1 & Butane & 2.8165 & 2.6667 & 10 & 8 \\
\hline 2 & 2-Methyl propane & 2.598 & 2.25 & 12 & 4 \\
\hline 3 & Pentane & 2.9428 & 3.3333 & 14 & 12 \\
\hline 4 & 2-Methyl butane & 3.644 & 3.3667 & 16 & 14 \\
\hline 5 & 2,2-Dimethyl propane & 3.5777 & 3.2 & 20 & 16 \\
\hline 6 & Hexane & 4.633 & 4.3333 & 18 & 16 \\
\hline 7 & 2-Methyl pentane & 4.644 & 4.3667 & 20 & 18 \\
\hline 8 & 3-Methyl pentane & 4.69 & 4.4833 & 20 & 19 \\
\hline 9 & 2,2-Methyl butane & 4.6545 & 4.4 & 24 & 22 \\
\hline 10 & 2,3-Dimethyl butane & 4.6888 & 4.5 & 22 & 21 \\
\hline 11 & Heptane & 5.633 & 5.3333 & 22 & 20 \\
\hline 12 & 2-Methyl hexane & 5.644 & 5.3667 & 24 & 22 \\
\hline 13 & 3-Methyl hexane & 5.6899 & 5.4833 & 24 & 23 \\
\hline 14 & 3-Ethyl pentane & 5.7358 & 5.6 & 24 & 24 \\
\hline 15 & 2,2-Dimethyl pentane & 5.6544 & 5.4 & 28 & 26 \\
\hline 16 & 2,3-Dimethyl pentane & 5.7347 & 5.616 & 26 & 26 \\
\hline 17 & 2,4-Dimethyl pentane & 5.655 & 5.4 & 26 & 24 \\
\hline 18 & 3,3-Dimethyl pentane & 5.7312 & 5.6 & 28 & 28 \\
\hline 19 & Octane & 6.633 & 6.333 & 26 & 24 \\
\hline 20 & 2-Methyl heptane & 6.644 & 6.3667 & 28 & 26 \\
\hline 21 & 3-Methyl heptane & 6.69 & 6.4833 & 28 & 27 \\
\hline 22 & 4-Methyl heptane & 6.68 & 6.49 & 28 & 27 \\
\hline 23 & 3-Ethyl hexane & 6.736 & 6.6 & 28 & 28 \\
\hline 24 & 2,2-Dimethyl hexane & 6.6545 & 6.4 & 32 & 30 \\
\hline 25 & 2,3-Dimethyl hexane & 6.7348 & 6.6166 & 30 & 30 \\
\hline 26 & 2,4-Dimethyl hexane & 6.7009 & 6.5166 & 30 & 29 \\
\hline 27 & 2,5-Dimethyl hexane & 6.655 & 6.4 & 32 & 30 \\
\hline 28 & 3,3-Dimethyl hexane & 6.7312 & 6.6 & 32 & 32 \\
\hline 29 & 3,4-Dimethyl hexane & 6.7807 & 6.7333 & 30 & 31 \\
\hline 30 & 3-Ethyl-2-methyl pentane & 6.7807 & 6.7333 & 30 & 22 \\
\hline 31 & 3-Ethyl-3-methyl pentane & 6.808 & 6.8 & 32 & 34 \\
\hline 32 & 2,2,3-Trimethyl pentane & 6.7706 & 6.731 & 34 & 35 \\
\hline 33 & $2,2,4$-Trimethyl pentane & 6.6655 & 6.4333 & 34 & 32 \\
\hline 34 & 2,3,3-Trimethyl pentane & 6.8014 & 6.8143 & 34 & 36 \\
\hline 35 & $2,3,4$-Trimethyl pentane & 6.7796 & 6.75 & 32 & 33 \\
\hline 36 & Nonane & 7.6333 & 7.3333 & 30 & 28 \\
\hline 37 & 2-Methyl octane & 7.644 & 7.3667 & 32 & 30 \\
\hline 38 & 3-Methyl octane & 7.69 & 7.4833 & 32 & 31 \\
\hline 39 & 4-Methyl octane & 7.69 & 7.4833 & 32 & 31 \\
\hline 40 & 3-Ethyl heptane & 7.7358 & 7.6 & 32 & 32 \\
\hline 41 & 4-Ethyl heptane & 7.7358 & 7.6 & 32 & 32 \\
\hline 42 & 2,2-Dimethyl heptane & 7.6545 & 7.4 & 36 & 34 \\
\hline 43 & 2,3-Dimethyl heptane & 7.7348 & 7.6166 & 34 & 34 \\
\hline 44 & 2,4-Dimethyl heptane & 7.7009 & 7.5166 & 34 & 33 \\
\hline 45 & 2,5-Dimethyl heptane & 7.7963 & 7.7166 & 34 & 33 \\
\hline 46 & 2,6-Dimethyl heptane & 7.655 & 7.4 & 34 & 32 \\
\hline 47 & 3,3-Dimethyl heptane & 7.7312 & 7.6 & 36 & 36 \\
\hline 48 & 3,4-Dimethyl heptane & 7.7807 & 7.7333 & 34 & 35 \\
\hline 49 & 3,5-Dimethyl heptane & 7.7468 & 7.6333 & 34 & 34 \\
\hline 50 & 4,4-Dimethyl heptane & 7.7312 & 7.6 & 36 & 36 \\
\hline 51 & 3-Ethyl-2-methyl hexane & 7.7807 & 7.7333 & 34 & 35 \\
\hline 52 & 4-Ethyl-2-methyl hexane & 7.9246 & 8.0333 & 34 & 34 \\
\hline 53 & 3-Ethyl-3-methyl hexane & 7.808 & 7.8 & 36 & 36 \\
\hline 54 & 2,2,4-Trimethyl hexane & 7.7114 & 7.55 & 38 & 37 \\
\hline 55 & 2,2,5-Trimethyl hexane & 7.6655 & 7.4333 & 38 & 36 \\
\hline 56 & 2,3,3-Trimethyl hexane & 7.8014 & 7.8143 & 38 & 40 \\
\hline 57 & 2,3,4-Trimethyl hexane & 7.8255 & 7.8667 & 36 & 44 \\
\hline 58 & 2,3,5-Trimethyl hexane & 7.7458 & 7.65 & 36 & 44 \\
\hline 59 & 3,3,4-Trimethyl hexane & 7.8845 & 8 & 38 & 41 \\
\hline 60 & 3,3-Diethyl pentane & 7.866 & 7.931 & 44 & 40 \\
\hline
\end{tabular}


TABle 1: Continued.

\begin{tabular}{|c|c|c|c|c|c|}
\hline SI. No. & Alkane & SS (G) & ISI (G) & $M_{1}(G)$ & $M_{2}(G)$ \\
\hline 61 & 2,2-Dimethyl-3-ethyl pentane & 7.878 & 8.0143 & 54 & 40 \\
\hline 62 & 2,3-Dimethyl-3-ethyl pentane & 7.8255 & 7.8666 & 46 & 42 \\
\hline 63 & 2,4-Dimethyl-3-ethyl pentane & 7.8575 & 8 & 44 & 38 \\
\hline 64 & 2,2,3,3-Tetramethyl pentane & 7.8575 & 8 & 42 & 46 \\
\hline 65 & 2,2,3,4-Tetramethyl pentane & 7.8154 & 7.643 & 40 & 42 \\
\hline 66 & 2,2,4,4-Tetramethyl pentane & 7.676 & 7.4667 & 42 & 40 \\
\hline 67 & 2,3,3,4-Tetramethyl pentane & 7.8715 & 8.0281 & 40 & 44 \\
\hline
\end{tabular}

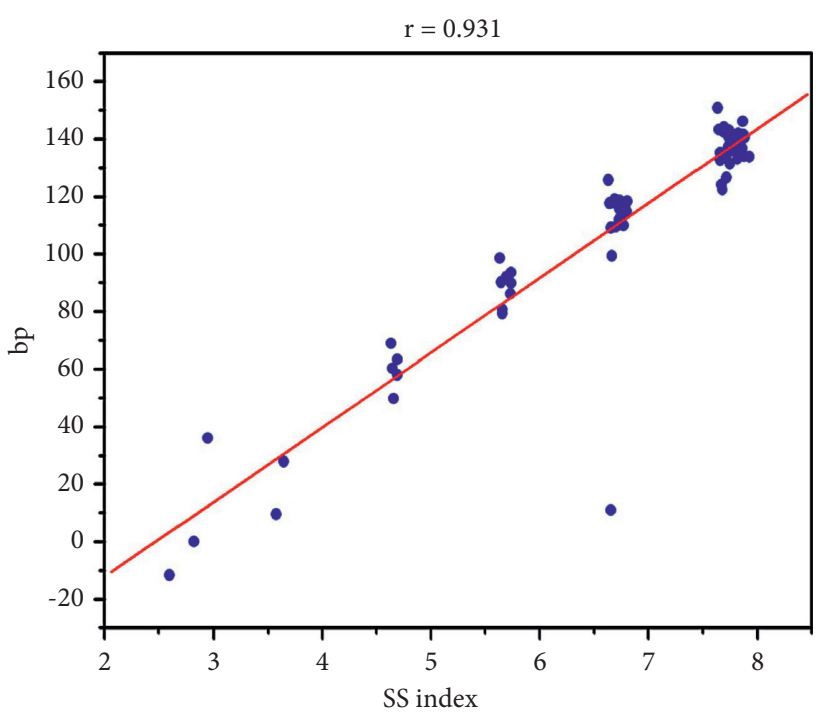

(a)

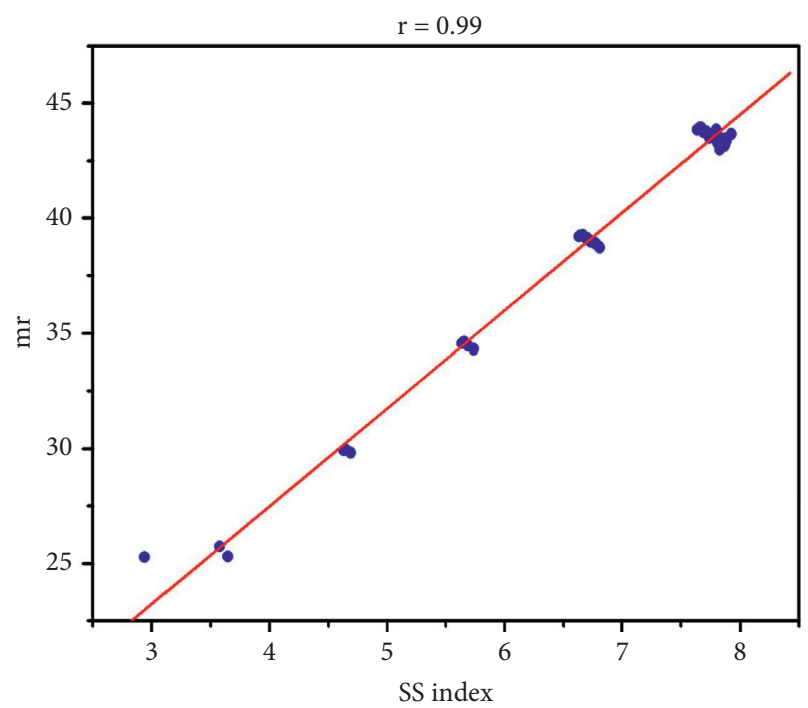

(c)

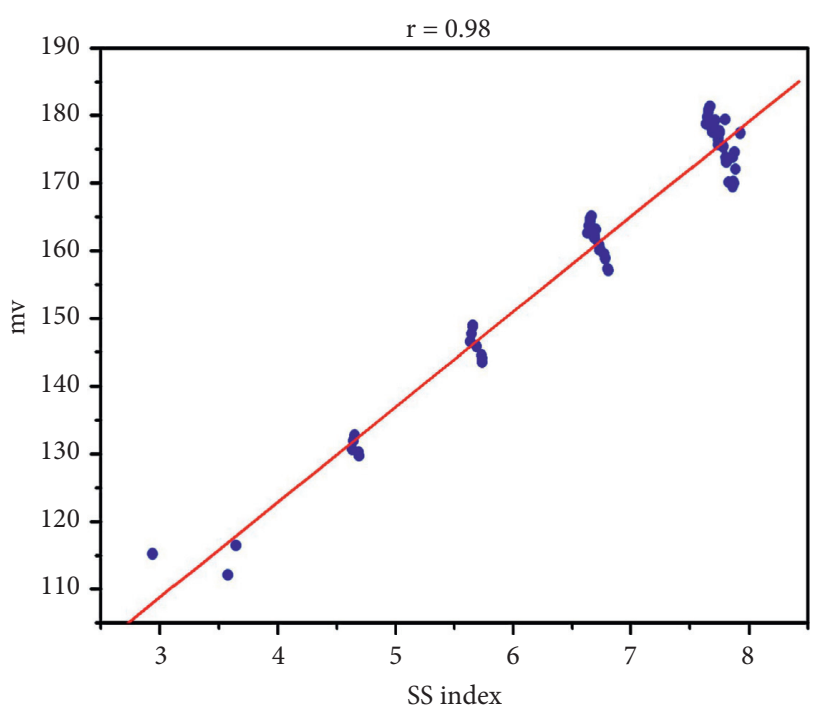

(b)

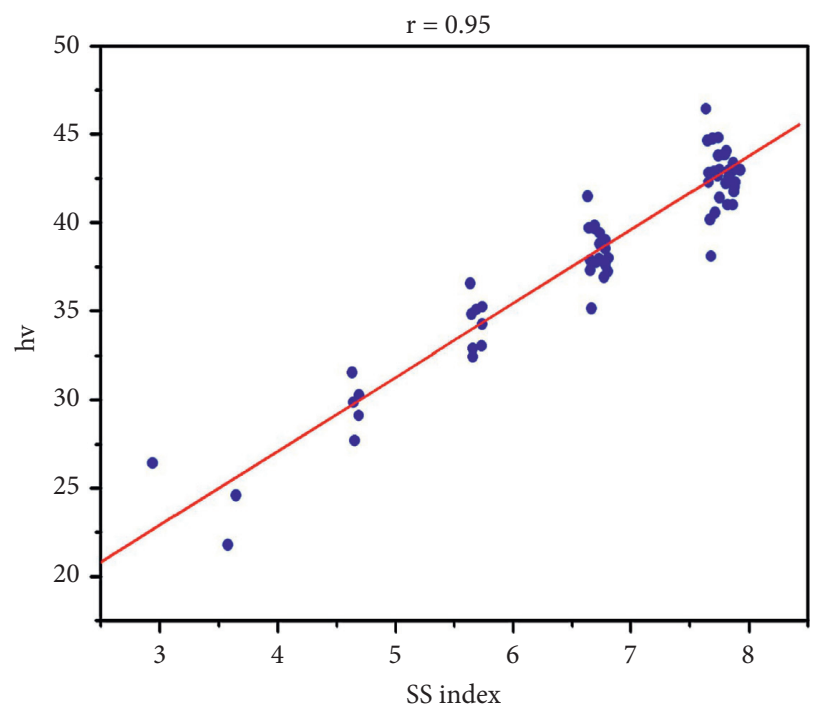

(d)

Figure 1: Continued. 


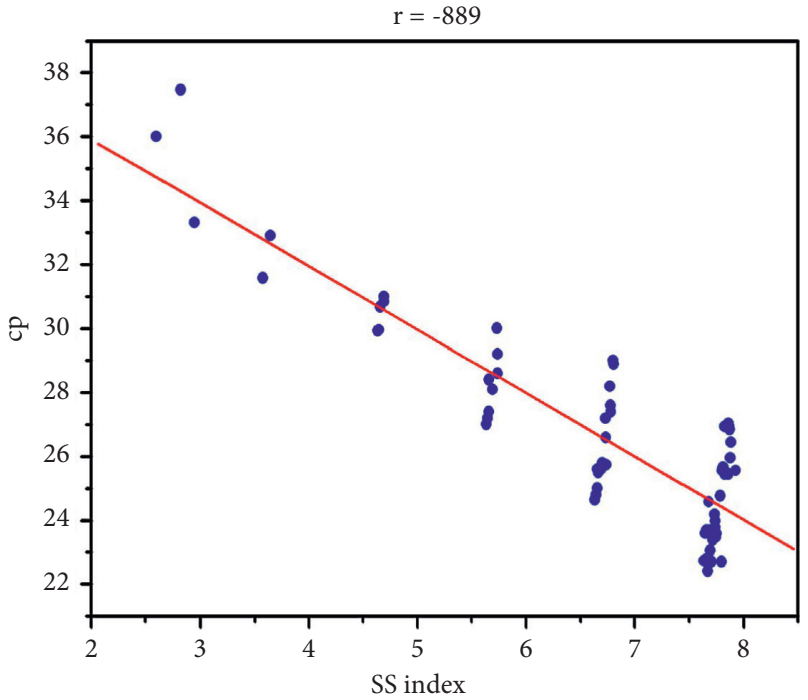

(e)

FIGURE 1: Correlation of SS index with properties of alkane isomers.

$$
\begin{aligned}
b p & =-64.28+25.99(\mathrm{SS}), \\
m v & =66.57+14.07(\mathrm{SS}), \\
m r & =10.46+4.26(\mathrm{SS}), \\
h v & =10.4+4.173(\mathrm{SS}), \\
c p & =39.87-1.98(\mathrm{SS}) .
\end{aligned}
$$

The SS index has correlation coefficients of $0.931,0.98$, $0.99,0.951$, and -0.889 with residual standard errors 14.46 , $3.76,0.57,1.69$, and 1.45 and all these models are statistically significant, since the level of significance value of all models is less than 0.05 .

Some important observations from the data are presented in Table 2. The correlation coefficients of $b p, m v, m r$, and $h v$ have shown high positive correlation for the introduced SS index. Also, it is interesting to know that the correlation coefficient for $c p$ shows highly negative correlation for the SS index.

\section{Dendrimers}

Dendrimers, come from the Greek word which means "trees," are branched at the core and they form a spherical three-dimensional structure. Dendrimers have attracted a lot of researchers globally in the study of topological indices
TABLE 2: The correlation coefficient between SS, ISI, $M_{1}$, and $M_{2}$ indices with some physico-chemical properties of alkane isomers.

\begin{tabular}{lccccc}
\hline Properties & $b p$ & $m v$ & $m r$ & $h v$ & $c p$ \\
\hline SS (G) & 0.931 & 0.98 & 0.99 & 0.951 & -0.889 \\
ISI (G) & 0.931 & 0.96 & 0.984 & 0.942 & -0.855 \\
$M_{1}$ (G) & 0.779 & 0.799 & 0.837 & 0.715 & -0.684 \\
$M_{2}(\mathrm{G})$ & 0.816 & 0.8065 & 0.854 & 0.737 & -0.688 \\
\hline
\end{tabular}

[28-33]. The aim of this paper is to compute SS index of four dendrimer structures, namely, $D_{n} P_{n}, \mathrm{DPZ}_{n}, \mathrm{PETIM}$, and PETAA.

3.1. SS Index of Porphyrin Dendrimer $\left(D_{n} P_{n}\right)$. Consider the porphyrin dendrimer family. This family of dendrimers is denoted by $D_{n} P_{n}$. The molecular graph of $D_{n} P_{n}$ is shown in Figure 2.

Let $G$ be the molecular graph of $D_{n} P_{n}$. By calculation, it is found that $G$ consists of number of vertices and edges to be $96 n-10$ and $105 n-11$, respectively. Table 3 shows the six forms of edges in $D_{n} P_{n}(G)$ based on degrees of end vertices of each edge.

Theorem 1. Let $D_{n} P_{n}$ be the family of porphyrin dendrimers. Then, the SS index of $D_{n} P_{n}$ is given by

$$
\operatorname{SS}\left(D_{n} P_{n}\right)=\left[2\left(\frac{3}{4}\right)^{1 / 2}+24\left(\frac{4}{5}\right)^{1 / 2}+10+48\left(\frac{6}{5}\right)^{1 / 2}+13\left(\frac{9}{6}\right)^{1 / 2}+8\left(\frac{12}{7}\right)^{1 / 2}\right] n-\left[5+6\left(\frac{6}{5}\right)^{1 / 2}\right]
$$

Proof. From the definition of SS index and Table 3, we deduce 


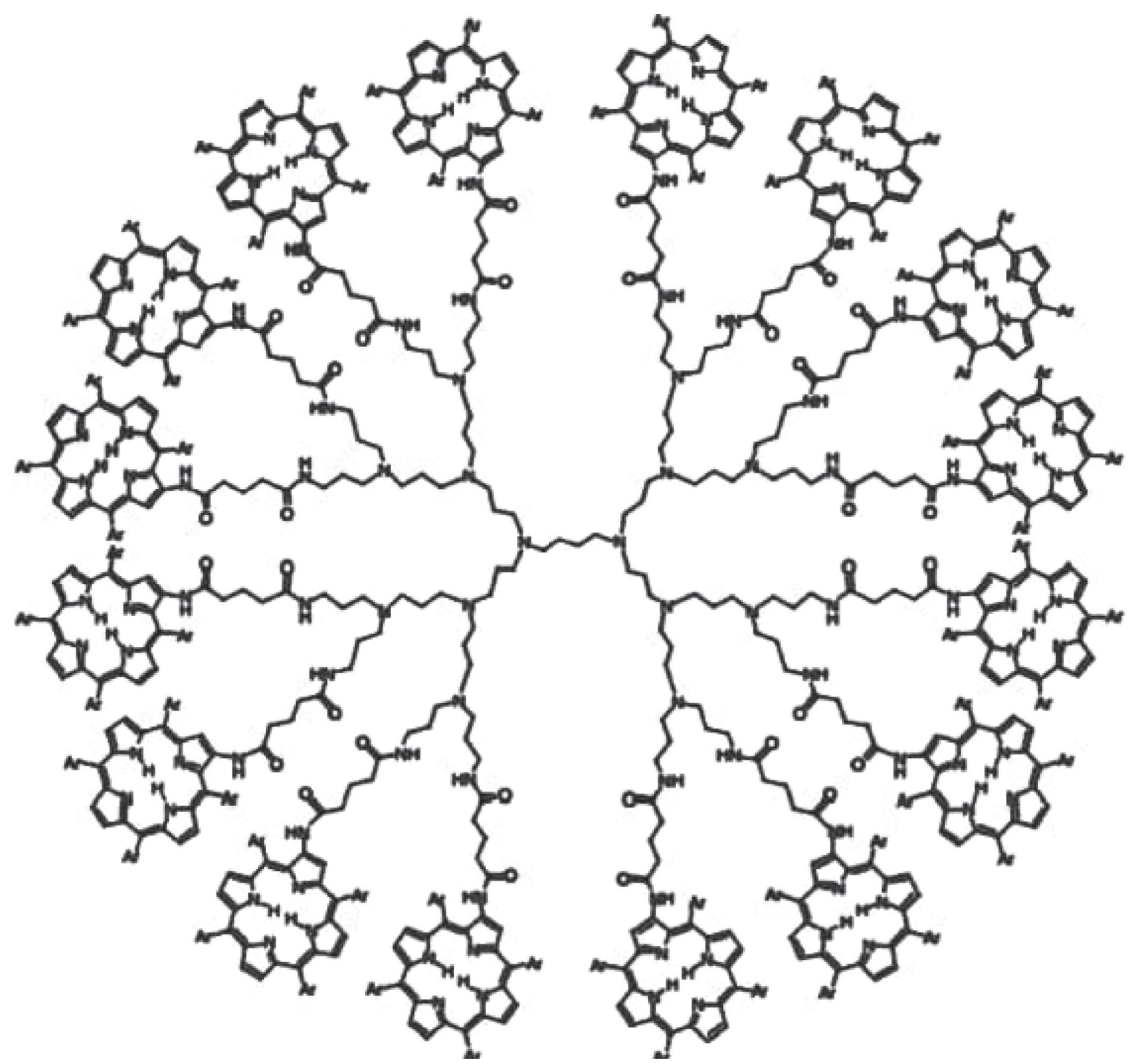

Figure 2: The molecular graph of porphyrin dendrimer.

TABle 3: Edge partition of $D_{n} P_{n}$.

\begin{tabular}{lcccccc}
\hline$\left(\mathrm{d}_{\nu}, \mathrm{d}_{\omega}\right)$, where $\nu \omega \in E(G)$ & $(1,3)$ & $(1,4)$ & $(2,2)$ & $(2,3)$ & $(3,3)$ & $(3,4)$ \\
\hline Number of edges & $2 n$ & $24 n$ & $10 n-5$ & $48 n-6$ & $13 n$ & $8 n$ \\
\hline
\end{tabular}

$$
\begin{aligned}
\operatorname{SS}\left(D_{n} P_{n}\right)= & \sum_{\nu \omega \in E(G)} \sqrt{\frac{\mathrm{d}_{\nu} \mathrm{d}_{\omega}}{\mathrm{d}_{\nu}+\mathrm{d}_{\omega}}}=2 n\left(\frac{1 \times 3}{1+3}\right)^{1 / 2}+24 n\left(\frac{1 \times 4}{1+4}\right)^{1 / 2}+(10 n-5)\left(\frac{2 \times 2}{2+2}\right)^{1 / 2}+(48 n-6)\left(\frac{2 \times 3}{2+3}\right)^{1 / 2} \\
& +13 n\left(\frac{3 \times 3}{3+3}\right)^{1 / 2}+8 n\left(\frac{3 \times 4}{3+4}\right)^{1 / 2}, \\
\operatorname{SS}\left(D_{n} P_{n}\right)= & {\left[2\left(\frac{3}{4}\right)^{1 / 2}+24\left(\frac{4}{5}\right)^{1 / 2}+10+48\left(\frac{6}{5}\right)^{1 / 2}+13\left(\frac{9}{6}\right)^{1 / 2}+8\left(\frac{12}{7}\right)^{1 / 2}\right] n-\left[5+6\left(\frac{6}{5}\right)^{1 / 2}\right] }
\end{aligned}
$$

3.2. SS Index of Zinc Porphyrin Dendrimer $\left(D P Z_{n}\right)$. Consider the zinc porphyrin dendrimer family. This family of dendrimers is represented by $\mathrm{DPZ} Z_{n}$. The molecular graph of $\mathrm{DPZ}_{n}$ is depicted in Figure 3.
Let $G$ be the molecular graph of $\mathrm{DPZ}_{n}$. By calculation, it is found that $G$ has $56 \times 2^{n}-7$ vertices and $64 \times 2^{n}-4$ edges. Table 4 shows the four forms of edges in $\mathrm{DPZ}_{n}$ based on degrees of end vertices of each edge. 


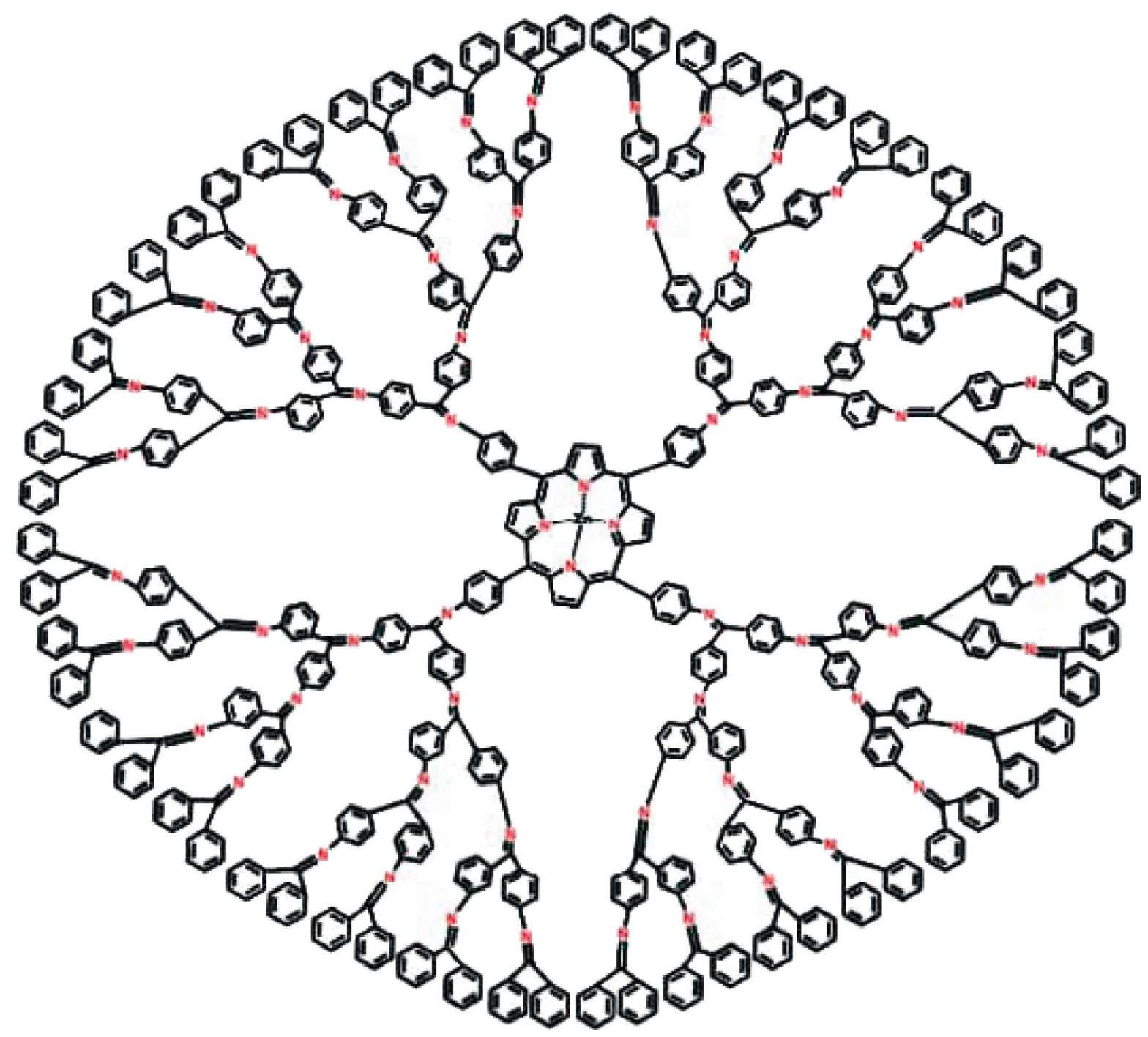

Figure 3: The molecular graph of zinc porphyrin dendrimer.

TABLE 4: Edge partition of $\mathrm{DPZ}_{n}$

\begin{tabular}{|c|c|c|c|c|}
\hline$\left(\mathrm{d}_{\nu}, \mathrm{d}_{\omega}\right)$, where $\nu \omega \in E(G)$ & $(2,2)$ & $(2,3)$ & $(3,3)$ & $(3,4)$ \\
\hline Number of edges & $16 \times 2^{n}-4$ & $40 \times 2^{n}-16$ & $8 \times 2^{n}-16$ & 4 \\
\hline
\end{tabular}

Theorem 2. Let $D P Z_{n}$ be the family of zinc porphyrin dendrimers. Then, the SS index of $\mathrm{DPZ}_{n}$ is given by

$$
\operatorname{SS}\left(\mathrm{DPZ}_{n}\right)=\left[16+40\left(\frac{6}{5}\right)^{1 / 2}+8\left(\frac{9}{6}\right)^{1 / 2}\right] 2^{n}-\left[4+16\left(\frac{16}{5}\right)^{1 / 2}+16\left(\frac{9}{6}\right)^{1 / 2}-4\left(\frac{12}{7}\right)^{1 / 2}\right]
$$

Proof. From the definition of SS index and Table 4, we deduce 


$$
\begin{aligned}
\mathrm{SS}\left(\mathrm{DPZ}_{n}\right)= & \sum_{\nu \omega \in E(G)} \sqrt{\frac{\mathrm{d}_{\nu} \mathrm{d}_{\omega}}{\mathrm{d}_{\nu}+\mathrm{d}_{\omega}}}=\left(16 \times 2^{n}-4\right)\left(\frac{2 \times 2}{2+2}\right)^{1 / 2}+\left(40 \times 2^{n}-16\right)\left(\frac{2 \times 3}{2+3}\right)^{1 / 2} \\
& +\left(8 \times 2^{n}-16\right)\left(\frac{3 \times 3}{3+3}\right)^{1 / 2}+4\left(\frac{3 \times 4}{3+4}\right)^{1 / 2}, \\
\mathrm{SS}\left(\mathrm{DPZ}_{n}\right)= & {\left[16+40\left(\frac{6}{5}\right)^{1 / 2}+8\left(\frac{9}{6}\right)^{1 / 2}\right] 2^{n}-\left[4+16\left(\frac{16}{5}\right)^{1 / 2}+16\left(\frac{9}{6}\right)^{1 / 2}-4\left(\frac{12}{7}\right)^{1 / 2}\right] . }
\end{aligned}
$$

3.3. SS Index of Propyl Ether Imine Dendrimer (PETIM). Consider the family of propyl ether imine dendrimers. This family of dendrimers is represented by PETIM. The molecular graph of PETIM is depicted in Figure 4.

Let $G$ be the molecular graph of PETIM. By calculation, $G$ has $24 \times 2^{n}-23$ vertices and $24 \times 2^{n}-24$ edges. Table 5 shows the three forms of edges in PETIM based on degrees of end vertices of each edge.
Theorem 3. Let PETIM be the family of propyl ether imine dendrimers. Then, the SS index of PETIM is given by

$$
\mathrm{SS}(\mathrm{PETIM})=\left[2\left(\frac{2}{3}\right)^{1 / 2}+16+6\left(\frac{6}{5}\right)^{1 / 2}\right] 2^{n}-\left[18+6\left(\frac{6}{5}\right)^{1 / 2}\right] \text {. }
$$

Proof. From the definition of SS index and Table 5, we deduce

$$
\begin{aligned}
& S S(\text { PETIM })=\sum_{\nu \omega \in E(G)} \sqrt{\frac{\mathrm{d}_{v} \mathrm{~d}_{\omega}}{\mathrm{d}_{v}+\mathrm{d}_{\omega}}}=\left(2 \times 2^{n}\right)\left(\frac{1 \times 2}{1+2}\right)^{1 / 2}+\left(16 \times 2^{n}-18\right)\left(\frac{2 \times 2}{2+2}\right)^{1 / 2}+\left(6 \times 2^{n}-6\right)\left(\frac{2 \times 3}{2+3}\right)^{1 / 2}, \\
& S S(P E T I M)=\left[2\left(\frac{2}{3}\right)^{1 / 2}+16+6\left(\frac{6}{5}\right)^{1 / 2}\right] 2^{n}-\left[18+6\left(\frac{6}{5}\right)^{1 / 2}\right] .
\end{aligned}
$$

3.4. SS Index of Polyethylene Amide Amine (PETAA) Dendrimer. Consider the family of polyethylene amide amine dendrimers. This family of dendrimers is represented by PETAA. The molecular graph of PETAA is depicted in Figure 5.

Let $G$ be the molecular graph of PETAA. By calculation, $G$ has $44 \times 2^{n}-18$ vertices and $44 \times 2^{n}-19$ edges. Table 6 shows the four forms of edges in PETAA based on degrees of end vertices of each edge.

Theorem 4. Let PETAA be the family of zinc porphyrin dendrimers. Then, the SS index of PETAA is given by

$$
\operatorname{SS}(\text { PETAA })=\left[4\left(\frac{2}{3}\right)^{1 / 2}+4\left(\frac{3}{4}\right)^{1 / 2}+16+20\left(\frac{6}{5}\right)^{1 / 2}\right] 2^{n}-\left[2\left(\frac{3}{4}\right)^{1 / 2}+8+9\left(\frac{6}{5}\right)^{1 / 2}\right]
$$

Proof. From the definition of SS index and Table 6, we deduce

$$
\begin{aligned}
\operatorname{SS}(\text { PETAA })= & \sum_{\nu \omega \in E(G)} \sqrt{\frac{\mathrm{d}_{\nu} \mathrm{d}_{\omega}}{\mathrm{d}_{v}+\mathrm{d}_{\omega}}}=\left(4 \times 2^{n}\right)\left(\frac{1 \times 2}{1+2}\right)^{1 / 2}+\left(4 \times 2^{n}-2\right)\left(\frac{1 \times 3}{1+3}\right)^{1 / 2}+\left(16 \times 2^{n}-8\right)\left(\frac{2 \times 2}{2+2}\right)^{1 / 2} \\
& +\left(20 \times 2^{n}-9\right)\left(\frac{2 \times 3}{2+3}\right)^{1 / 2}, \\
\operatorname{SS}(\text { PETAA })= & {\left[4\left(\frac{2}{3}\right)^{1 / 2}+4\left(\frac{3}{4}\right)^{1 / 2}+16+20\left(\frac{6}{5}\right)^{1 / 2}\right] 2^{n}-\left[2\left(\frac{3}{4}\right)^{1 / 2}+8+9\left(\frac{6}{5}\right)^{1 / 2}\right] }
\end{aligned}
$$




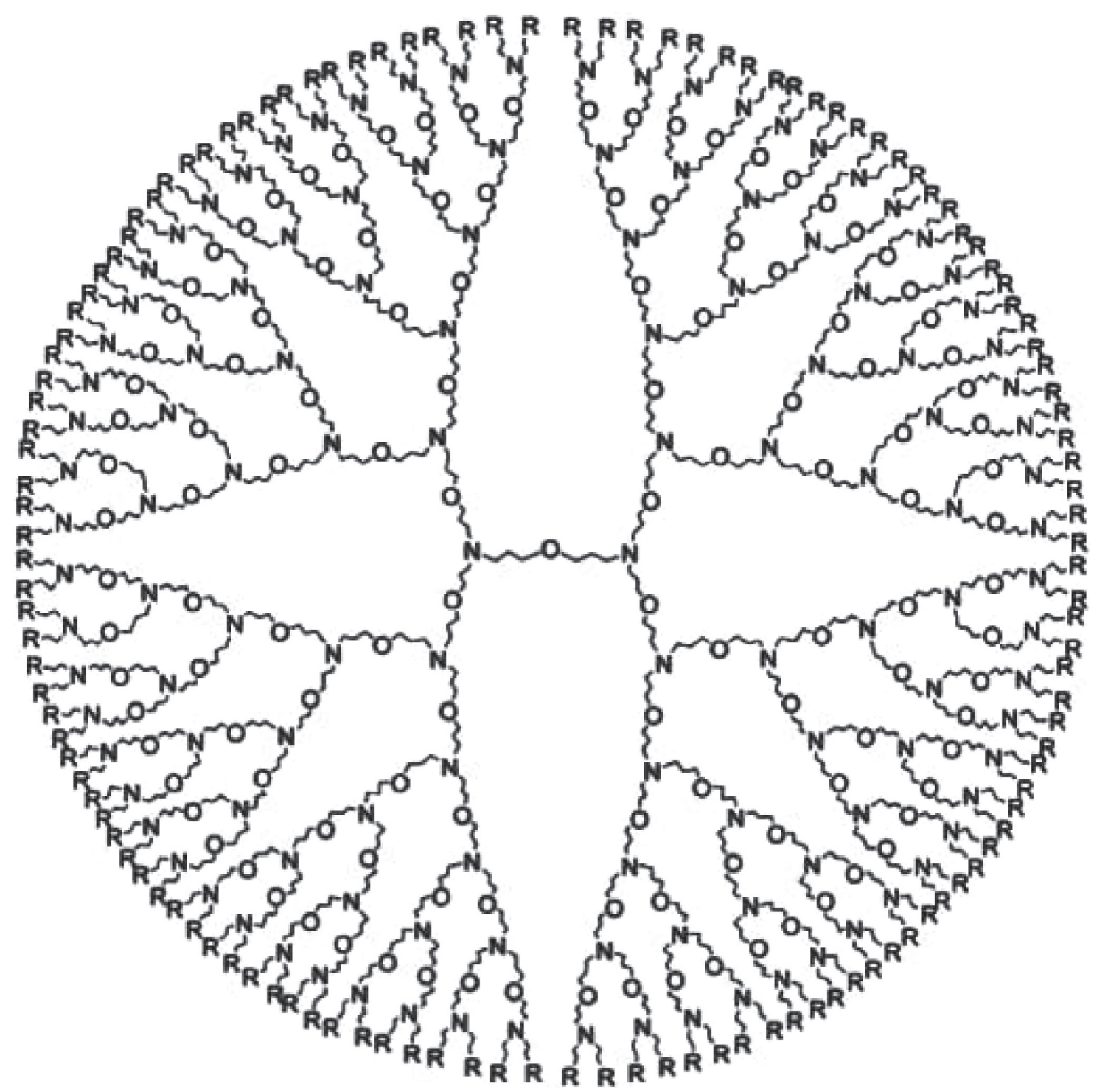

FIgURE 4: The molecular graph of propyl ether imine dendrimer.

TABle 5: Edge partition of PETIM.

\begin{tabular}{|c|c|c|c|}
\hline$\left(\mathrm{d}_{\nu}, \mathrm{d}_{\omega}\right)$, where $\nu \omega \in E(G)$ & $(1,2)$ & $(2,2)$ & $(2,3)$ \\
\hline Number of edges & $2 \times 2^{n}$ & $16 \times 2^{n}-18$ & $6 \times 2^{n}-6$ \\
\hline
\end{tabular}

\section{Results and Discussion}

In this work, novel topological index known as SS index is introduced and the proposed index is computed for 67 alkane isomers to study the physico-chemical properties, namely, $b p, m v, m r, h v$, and $c p$. A linear regression model of these physical properties with SS index is presented. From Table 2 and Figure 1, the SS index has highest correlation with molar refraction $(m r)$ which is 0.99 . Also, SS index is with boiling point $(b p)$ 0.931, with molar volume $(m v)$ 0.98, with heat of vaporization $(h v) 0.951$, and with critical pressure $(c p)-0.889$. From Table 2 by inspection, it is clear that SS index has good correlation with the physico-chemical properties compared to the existing indices, namely, inverse sum indeg index, first Zagreb index, and second Zagreb index. Also, the work focuses on computing the SS index for four dendrimer structures, namely, $D_{n} P_{n}, \mathrm{DPZ}_{n}, \mathrm{PETIM}$, and PETAA. The values of $n$ are substituted for $n=1$ to 10 . By inspection from Table 7 , it is very clear that SS index increases as $n$ increases. Also, it is observed that correlation coefficient of $D_{n} P_{n}$ is $r=1$, which is more than the correlation coefficients of $\mathrm{DPZ}_{n}$, PETIM, and PETAA which are $0.798837,0.798841$, and 0.798835 , respectively. For each of the four structures, a graph as shown in Figure 6 is plotted against the values found in Table 7. 


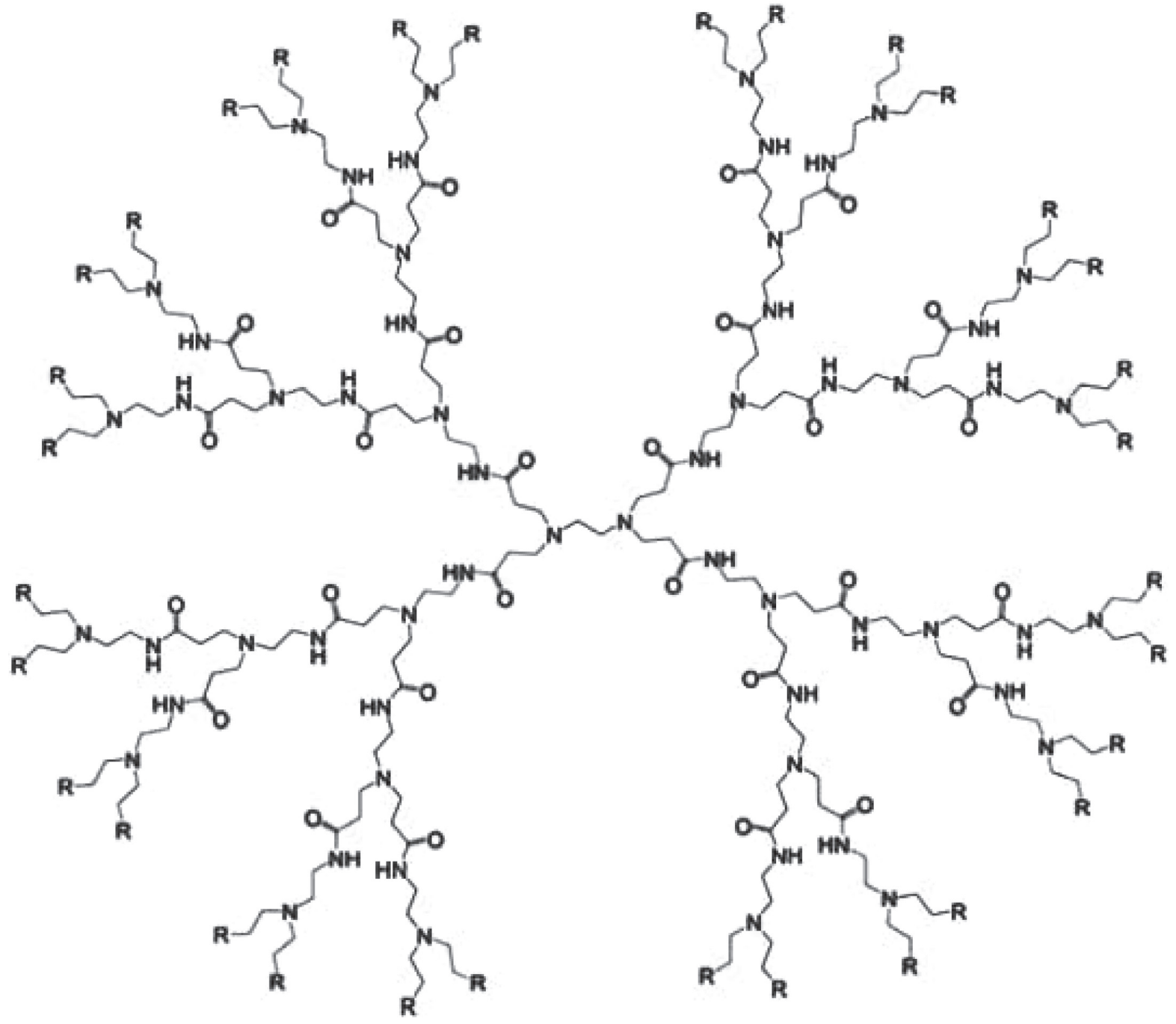

FIgURE 5: The molecular graph of polyethylene amide amine dendrimer.

TABle 6: Edge partition of PETAA.

\begin{tabular}{lcccc}
\hline $\begin{array}{l}\left(\mathrm{d}_{v}, \mathrm{~d}_{\omega}\right), \text { where } \\
\nu \omega \in E(G)\end{array}$ & $(1,2)$ & $(1,3)$ & $(2,2)$ & $(2,3)$ \\
\hline Number of edges & $4 \times 2^{n}$ & $4 \times 2^{n}-2$ & $16 \times 2^{n}-8$ & $20 \times 2^{n}-9$ \\
\hline
\end{tabular}

As the SS index is found to have very good correlation coefficient $r=0.99 \cong 1$ with the above-discussed physicochemical properties, the novel index is of great use in the QSPR/QSAR/QSTR analysis by the chemists.

\section{Mathematical Properties of SS Index}

In this section, the SS indexes of cycle, star, path, and simple graphs are computed [34-38].

Theorem 5. For a cycle $C_{n}$, where $n$ is the cardinality of vertices, then the SS index of $C_{n}$ is given by $S S\left(C_{n}\right)=n$.
Proof. A cycle $C_{n}$ has $n$ vertices and $n$ edges. The $n$ edges of the cycle will be of type $(2,2)$. By considering all the $n$ edges and using the definition of SS index, we get $\operatorname{SS}\left(C_{n}\right)=n$.

Theorem 6. For a star $S_{n}$, where $n$ is the cardinality of vertices, then the SS index of $S_{n}$ is given by

$$
\operatorname{SS}\left(S_{n}\right)=\frac{(n-1)^{3 / 2}}{\sqrt{n}} .
$$

Proof. A star $S_{n}$ has $n$ vertices and $(n-1)$ edges. The $(n-1)$ edges of the star graph will be of type $(1, n-1)$. By considering all the $(n-1)$ edges and using the definition of SS index, we get

$$
\operatorname{SS}\left(S_{n}\right)=\frac{(n-1)^{3 / 2}}{\sqrt{n}} .
$$


TABLE 7: Numerical comparison of SS $\left(D_{n} P_{n}\right), \operatorname{SS}\left(\mathrm{DPZ}_{n}\right)$, SS(PETIM), and SS(PETAA) for $n=1$ to 10.

\begin{tabular}{|c|c|c|c|c|c|c|c|c|c|c|}
\hline$n$ & 1 & 2 & 3 & 4 & 5 & 6 & 7 & 8 & 9 & 10 \\
\hline$D_{n} P_{n}$ & 100.60 & 212.78 & 324.95 & 437.13 & 549.31 & 661.48 & 773.66 & 885.84 & 998.01 & 1110.2 \\
\hline $\mathrm{DPZ}_{n}$ & 92.252 & 231.48 & 509.95 & 1066.9 & 2180.7 & 4408.4 & 8863.9 & 17775 & 35596 & 71240 \\
\hline PETIM & 23.84 & 72.251 & 169.07 & 362.72 & 750.02 & 1524.6 & 3073.8 & 6172.2 & 12369 & 24762 \\
\hline PETTA & 37.69 & 94.97 & 209.53 & 438.65 & 896.89 & 1813.4 & 3646.3 & 7312.2 & 14644 & 29308 \\
\hline
\end{tabular}

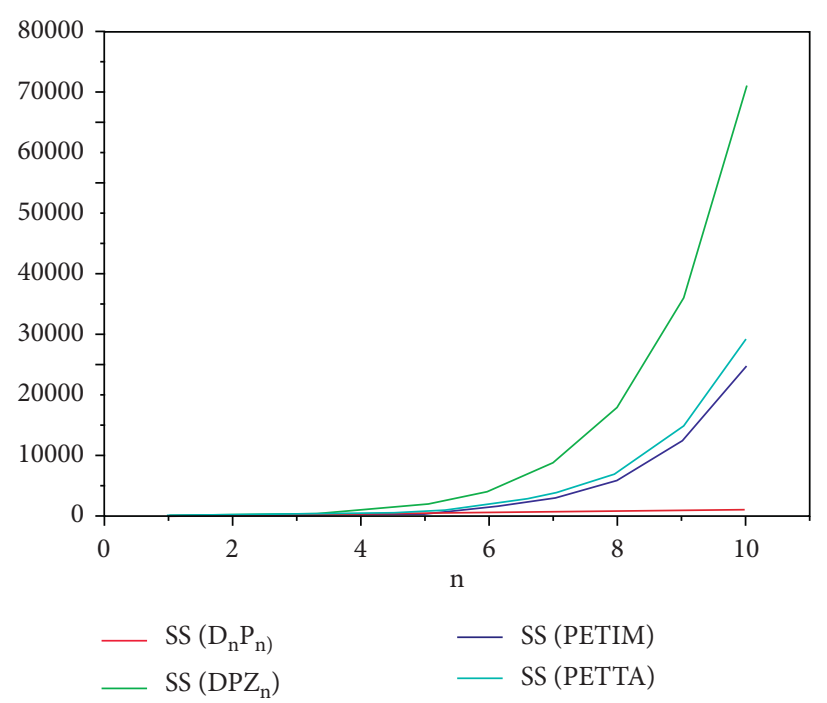

Figure 6: Graphical comparison of SS $\left(D_{n} P_{n}\right), \operatorname{SS}\left(\mathrm{DPZ}_{n}\right), \mathrm{SS}(\mathrm{PETIM})$, and SS(PETAA) for $n=1$ to 10.

Theorem 7. For a path $P_{n}$, where $n$ is the cardinality of vertices, then the SS index of $P_{n}$ is given by $S S\left(P_{n}\right)$ $=(n-3)+(2 \sqrt{2} / \sqrt{3})$.

Proof. A path $P_{n}$ has $n$ vertices and $(n-1)$ edges. The $(n-1)$ edges of the path graph will be 2 edges of type $(1,2)$ and $(n-3)$ edges of type $(2,2)$, respectively. By considering all the $(n-1)$ edges and using the definition of SS index, we get $\mathrm{SS}\left(P_{n}\right)=(n-3)+(2 \sqrt{2} / \sqrt{3})$.

The SS indexes of cycle, star, and path graphs are related as follows:

$$
\begin{gathered}
\operatorname{SS}(S)=\frac{(n-1)^{3 / 2}}{\sqrt{S S\left(C_{n}\right)}} \\
\operatorname{SS}\left(P_{n}\right)=\operatorname{SS}\left(C_{n}\right)+\frac{2 \sqrt{2}}{\sqrt{3}}-3 .
\end{gathered}
$$

$$
\begin{aligned}
\left(\sum_{v w \in E(G): d_{v}, d_{w} \neq 1} \sqrt{\frac{\mathrm{d}_{v} \mathrm{~d}_{\omega}}{\mathrm{d}_{v}+\mathrm{d}_{\omega}}}\right)^{2} \geq & \frac{4 n \delta_{1} \Delta}{\left(\Delta+\delta_{1}\right)^{2}}\left(\sum_{v w \in E(G): d_{v}, d_{w} \neq 1} \frac{\mathrm{d}_{\nu} \mathrm{d}_{\omega}}{\mathrm{d}_{v}+\mathrm{d}_{\omega}}\right) \geq \frac{4 n \delta_{1} \Delta}{\left(\Delta+\delta_{1}\right)^{2}}\left(\operatorname{ISI}(G)-\sum_{v w \in E(G): d_{v}=1} \frac{\mathrm{d}_{\omega}}{1+\mathrm{d}_{\omega}}\right) \\
& \left(\sum_{v w \in E(G): d_{v}, d_{w} \neq 1} \sqrt{\frac{\mathrm{d}_{\nu} \mathrm{d}_{\omega}}{\mathrm{d}_{v}+\mathrm{d}_{\omega}}}\right) \geq \frac{2 \sqrt{n \delta_{1} \Delta}}{\left(\Delta+\delta_{1}\right)} \sqrt{\operatorname{ISI}(G)-\frac{p \delta_{1}}{1+\delta_{1}}}
\end{aligned}
$$


we have

$$
\mathrm{SS}(G)=\sum_{\nu \omega \in E(G)} \sqrt{\frac{d_{w}}{1+d_{w}}}+\left(\sum_{\nu w \in E(G): d_{\nu}, d_{w} \neq 1} \sqrt{\frac{\mathrm{d}_{\nu} \mathrm{d}_{\omega}}{\mathrm{d}_{v}+\mathrm{d}_{\omega}}}\right) .
$$

For $\Delta \geq d_{v}$, from (20) and (21), we get

$$
\mathrm{SS}(G) \geq \frac{p \sqrt{\delta_{1}}}{\sqrt{1+\delta_{1}}}+\frac{2 \sqrt{n \delta_{1} \Delta}}{\Delta+\delta_{1}} \sqrt{I S I(G)-\frac{p \delta_{1}}{1+\delta_{1}}} .
$$

Theorem 10. Consider simple graph $G$ of order $n$ with $m$ edges and cardinality $n$. Here, $p, \Delta$, and $\delta_{1}$ represent pendent vertices and maximum vertex degree and minimum nonpendent vertex degree, respectively, and then

$$
\mathrm{SS}(G) \leq \frac{p \sqrt{\Delta}}{\sqrt{1+\Delta}}+\sqrt{(m-p)\left[\operatorname{ISI}(G)-\frac{p \Delta}{1+\Delta}\right]} .
$$

Proof. By Cauchy-Schwarz inequality,

Theorem 9. For a tree $T$ with cardinality $n$ and pendent vertices $p$, then the SS index is

$$
\mathrm{SS}(T) \geq \frac{p \sqrt{\delta_{1}}}{\sqrt{1+\delta_{1}}}+\frac{2 \sqrt{(m-1) \delta_{1} \Delta}}{\Delta+\delta_{1}} \sqrt{\operatorname{ISI}(G)-\frac{p \delta_{1}}{1+\delta_{1}}} .
$$

$$
\begin{aligned}
\left(\sum_{v w \in E(G): d_{v}, d_{w} \neq 1} \sqrt{\frac{\mathrm{d}_{\nu} \mathrm{d}_{\omega}}{\mathrm{d}_{v}+\mathrm{d}_{\omega}}}\right)^{2} & \leq(m-p)\left(\sum_{v w \in E(G): d_{v}, d_{w} \neq 1} \frac{\mathrm{d}_{v} \mathrm{~d}_{\omega}}{\mathrm{d}_{v}+\mathrm{d}_{\omega}}\right), \\
& \leq(m-p)\left[\operatorname{ISI}(G)-\sum_{v w \in E(G): d_{v}=1} \frac{\mathrm{d}_{\omega}}{1+\mathrm{d}_{\omega}}\right] \\
& \leq(m-p)\left[\operatorname{ISI}(G)-\frac{p \Delta}{1+\Delta}\right] \\
\left.\sum_{v w \in E(G): d_{v}, d_{w} \neq 1} \sqrt{\frac{\mathrm{d}_{\nu} \mathrm{d}_{\omega}}{\mathrm{d}_{v}+\mathrm{d}_{\omega}}}\right) & \leq \sqrt{(m-p)\left(\operatorname{ISI}(G)-\frac{p \Delta}{1+\Delta}\right)} \\
\operatorname{SS}_{(G)} & \leq \frac{p \sqrt{\Delta}}{\sqrt{1+\Delta}}+\sqrt{(m-p)\left[\operatorname{ISI}(G)-\frac{p \Delta}{1+\Delta}\right]}
\end{aligned}
$$

Theorem 11. The cardinality $n$ of a tree $T$ and pendent vertices $p$ and then the SS index is

$$
\mathrm{SS}(T) \leq \frac{p \sqrt{\Delta}}{\sqrt{1+\Delta}}+\sqrt{(n-1-p)\left[\operatorname{ISI}(G)-\frac{p \Delta}{1+\Delta}\right]} .
$$

\section{Conclusion}

In this article, a novel index known as SS index is introduced and computed for four dendrimers such as $D_{n} P_{n}, \mathrm{DPZ}_{\mathrm{n}}$, PETIM, and PETAA. To validate the performance of this novel index, the chemical applicability of 67 alkane isomers is studied. It is observed that the proposed index has a very good correlation with alkane isomers considered in the study. The results obtained for the dendrimers have proved that they play a major role in drugs including anti-inflammatory, antimicrobial, and anticancer in administering the drug. Dendrimers play a vital role in the discovery of drugs against the diseases such as Alzheimer's, HIV, and cancer. The article is concluded by mathematical properties of SS iindexes for cycle, star, path, and simple graphs.

\section{Data Availability}

No data were used to support this study.

\section{Conflicts of Interest}

The authors declare that they have no conflicts of interest.

\section{Authors' Contributions}

All the authors contributed equally to this study. M. C. Shanmukha gave the idea and wrote the manuscript. A. Usha and K. C. Shilpa edited and verified the results. Weidong Zhao checked and corrected the initial manuscript and verified the results. M. Reza Farahani added some final 
remarks and improved the overall paper. All authors read and approved the final draft.

\section{Acknowledgments}

This work was supported by the National Key Research and Development Program under Grant 2018YFB0904205. This research received no external funding.

\section{References}

[1] W. Gao, W. F. Wang, and L. Shi, "Forgotten topological of some drug structures," Acta Medica Mediterranea, vol. 32, pp. 579-585, 2016.

[2] M. C. Shanmukha, A. Usha, N. S. Basavarajappa, and K. C. Shilpa, "M-polynomials and topological indices of styrene-butadiene rubber (SBR)," Polycyclic Aromatic Compounds, vol. 6, pp. 1-16, 2020.

[3] N. Trinajstić, Chemical Graph Theory, CRC Press, Boca Raton, FL, USA, 1992.

[4] R. Todeschini and V. Consonni, Molecular Descriptors for Cheminformatics, Wiley VCH, Weinheim, Germany, 2009.

[5] W. Gao, M. K. Siddiqui, M. Imran, M. K. Jamil, and M. R. Farahani, "Forgotten topological index of chemical structure in drugs," Saudi Pharmaceutical Journal, vol. 24, no. 3, pp. 258-64, 2016.

[6] W. Gao, W. Wang, and M. R. Farahani, "Topological indices study of molecular structure in anticancer drugs," Journal of Chemistry, vol. 2016, Article ID 3216327, 8 pages, 2016.

[7] L. Yan, W. Gao, and J. Li, "General harmonic index and general sum connectivity index of polyomino chains and nanotubes," Journal of Computational and Theoretical Nanoscience, vol. 12, no. 10, pp. 3940-3944, 2015.

[8] E. Estrada, L. Torres, L. Roriguez, and I. Gutman, "An atombond connectivity index. Modelling the enthalpy of formation of alkanes," Indian Journal of Chemistry, vol. 37, pp. 849-855, 1998.

[9] V. Lokesha, A. Usha, P. S. Ranjini, and T. Deepika, "Hormonic index, redefined Zagreb indices of dragon graph with complete graph," Asian Journal of Mathematics and Computer Research, vol. 9, no. 2, pp. 161-166, 2016.

[10] P. S. Ranjini, V. Lokesha, and A. Usha, "Relation between phenylene and hexagonal squeez using harmonic index," International Journal of Graph Theory, vol. 1, pp. 116-121, 2013.

[11] M. C. Shanmukha, A. Usha, N. S. Basavarajappa, and K. C. Shilpa, "Graph entropies of porous graphene using topological indices," Computational and Theoretical Chemistry, vol. 1197, pp. 1-12, Article ID 113142, 2021.

[12] M. Randic, "Characterization of molecular branching," Journal of the American Chemical Society, vol. 97, no. 23, pp. 6609-6615, 1975.

[13] J. Zheng, S. Akhter, Z. Iqbal et al., "Irregularity measures of subdivision vertex-edge join of graphs," Journal of Chemistry, vol. 2021, Article ID 6673221, 12 pages, 2021.

[14] M. Randić, "Quantitative Structure property Relationship: boiling points and planar Benzenoid," New Journal of Chemistry, vol. 20, pp. 1001-1009, 1996.

[15] H. Wiener, "Structural determination of paraffin boiling points," Journal of the American Chemical Society, vol. 69, no. 1, pp. 17-20, 1947.

[16] I. Gutman and K. C. Das, "The first Zagreb index 30 years after," MATCH Communications in Mathematical and in Computer Chemistry, vol. 50, pp. 83-92, 2004.
[17] I. Gutman, B. Furtula, and C. Elphick, "Three new/old vertexdegree-based topological indices," MATCH Communications in Mathematical and in Computer Chemistry, vol. 72, no. 3, pp. 617-632, 2014.

[18] S. Li and M. Zhang, "Sharp upper bounds for Zagreb indices of bipartite graphs with a given diameter," Applied Mathematics Letters, vol. 24, no. 2, pp. 131-137, 2011.

[19] T. Reti, R. Sharafdini, A. Dregelyi-Kiss, and H. Haghbin, "Graph irregularity indices used as molecular descriptors in QSPR studies," MATCH Communications in Mathematical and in Computer Chemistry, vol. 79, pp. 509-524, 2018.

[20] V. Sharma, R. Goswami, and A. K. Madan, "Eccentric connectivity index: a novel highly discriminating topological descriptor for Structure-Property and Structure-Activity studies," Journal of Chemical Information and Computer Sciences, vol. 37, no. 2, pp. 273-282, 1997.

[21] B. Zhou and N. Trinajstić, "On general sum-connectivity index," Journal of Mathematical Chemistry, vol. 47, no. 1, pp. 210-218, 2010.

[22] V. R. Kulli, "Nirmala index," International Journal of Mathematics Trends and Technology, vol. 67, no. 3, pp. 8-12, 2021.

[23] W. Gao, M. Younas, A. Farooq, A.-R. Virk, and W. Nazeer, "Some reverse degree-based topological indices and polynomials of dendrimers," Mathematics, vol. 6, pp. 1-20, 2018.

[24] J. A. Bondy and U. S. R. Murty, Graph Theory, Springer, Berlin, Germany, 2008.

[25] I. Gutman and N. Trinajstić, "Graph theory and molecular orbitals. Total $\varphi$-electron energy of alternant hydrocarbons," Chemical Physics Letters, vol. 17, no. 4, pp. 535-538, 1972.

[26] D. Vukičević and M. Gasperov, "Bond aditive modeling 1. Adriatic indices," Croatica Chemica Acta, vol. 83, no. 3, pp. 243-260, 201.

[27] S. Mondal, A. Dey, N. De, and A. Pal, "QSPR analysis of some novel neighbourhood degree-based topological indices," Complex and Intelligent Systems, vol. 7, pp. 977-996, 2021.

[28] A. Ghorbani and M. A. Hosseinzadeh, "Computing ABC4 index of nanostar dendrimers," Optoelectronics and Advanced Materials-Rapid Communications, vol. 4, pp. 1419-1422, 2010.

[29] I. Graovac, M. Ghorbani, and M. A. Hosseinzadeh, "Computing fifth geometric arithmetic index for nanostar dendrimers," Journal of Mathematical Nanoscience, vol. 1, pp. 33-42, 2011.

[30] S. M. Kang, M. A. Zahid, A. U. R. Virk, W. Nazeer, and W. Gao, "Calculating the degree-based topological indices of dendrimers," Open Chemistry, vol. 16, no. 1, pp. 681-688, 2018.

[31] V. R. Kulli, B. Chaluvaraju, V. Lokesha, and S. A. Basha, "Gourava indices of some dendrimers," Research Review International Journal of Multidisciplinary, vol. 4, no. 6, pp. 212-215, 2019.

[32] M. Munir, W. Nazeer, S. Rafique, and S. Kang, "M-polynomial and related topological indices of nanostar dendrimers," Symmetry, vol. 8, no. 9, pp. 1-12, 2016.

[33] I. Goli Farkoush, M. Alaeiyan, M. R. Farahani, and M. Maghasedi, "Computing the narumi-katayama index and modified narumi-katayama index of some families of dendrimers and tetrathiafulvalene," Journal of Mathematics, vol. 2021, Article ID 8492991, 3 pages, 2021.

[34] C. Kinkar, "Atom-bond connectivity index of graphs," Discrete Applied Mathematics, vol. 158, no. 11, pp. 1181-1188, 2010.

[35] V. Lokesha, B. Shwetha Shetty, P. S. Ranjini, I. N. Cangul, and A. S. Cevik, "New bounds for Randic and GA indices," Journal 
of Inequalities and Applications, no. 1, pp. 1-7, Article ID 180, 2013.

[36] A. Usha, P. S. Ranjini, and Harish, "Bounds of harmonic index," Contemporary Studies in Discrete Mathematics, vol. 2, no. 1, pp. 27-29, 2018.

[37] P. -Szego, Problems and Theorems in Analysis I: Series, Integral Calculus, Theory of Functions, Springer, New York, NY, USA, 1972.

[38] K. Agilarasan and A. Selvakumar, "Some bounds on forgotten topological index," International Journal of Mathematics Trends and Technology, vol. 56, no. 7, pp. 1-3, 2018. 